\title{
Abbreviazioni
}

- Nelle citazioni di Aristotele si fa riferimento al testo di Bekker, salvo che nel caso di Hist. An., per la quale si tiene conto del testo di Dittmeyer 1907 e Balme 2002. Per Mir. ci si avvale invece dell'edizione critica ora in corso di pubblicazione.

$\begin{array}{llll}\text { Abbreviazioni (= ArGr) } & & \\ \text { An. } & \text { De anima } & \text { MM } & \text { Magna moralia } \\ \text { Anal. Post. } & \text { Analytica Posteriora } & \text { Mot. An. } & \text { De motu animalium } \\ \text { Anal. Pr. } & \text { Analytica Priora } & \text { Mu. } & \text { De mundo } \\ \text { Cael. } & \text { De caelo } & \text { MXG } & \text { De Melisso Xenophane Gorgia } \\ \text { Cat. } & \text { Categoriae } & \text { Oec. } & \text { Oeconomica } \\ \text { Col. } & \text { De coloribus } & \text { Part. An. } & \text { De partibus animalium } \\ \text { Div. Somn. } & \text { De divinatione per somnum } & \text { Phgn. } & \text { Physiognomonica } \\ \text { EE } & \text { Ethica ad Eudemum } & \text { Phys. } & \text { Physica } \\ \text { EN } & \text { Ethica Nicomachea } & \text { Plant. } & \text { De plantis } \\ \text { Gener. An. } & \text { De generatione animalium } & \text { Poet. } & \text { Poetica } \\ \text { Gener. Corr. } & \text { De generatione et corruptione } & \text { Pol. } & \text { Politica } \\ \text { Hist. An. } & \text { Historia animalium } & \text { Probl. } & \text { Problemata } \\ \text { Inc. An. } & \text { De incessu animalium } & \text { Respir. } & \text { De respiratione } \\ \text { Insomn. } & \text { De insomniis } & \text { Rhet. } & \text { Rhetorica } \\ \text { Int. } & \text { De interpretatione } & \text { Rhet. Al. } & \text { Rhetorica ad Alexandrum } \\ \text { Juv. } & \text { De iuventute (+ VM) } & \text { Sens. } & \text { De sensu } \\ \text { Lin. } & \text { De lineis insecabilibus } & \text { Somn. Vig. De somno et vigilia } \\ \text { Long. } & \text { De longitudine vitae } & \text { Soph. El. } & \text { Sophistici elenchi } \\ \text { Mech. } & \text { Mechanica } & \text { Spir. } & \text { De spiritu } \\ \text { Mem. } & \text { De memoria } & \text { Top. } & \text { Topica } \\ \text { Metaph. } & \text { Metaphysica } & \text { Vent. } & \text { De ventis } \\ \text { Mete. } & \text { Meteorologica } & \text { Virt. } & \text { De virtutibus et vitiis } \\ \text { Mir. } & \text { Mirabilia } & \text { VM } & \text { De vita et morte } \\ & & \end{array}$

Per il corpus teofrasteo, associato a Mir. nel ramo della tradizione dipendente dal Vat. gr. 1302 (= F), si fa riferimento alle seguenti edizioni:

De causis plantarum: Libri I-II: Amigues 2012 • Libri III-IV: Amigues 2015 • Libri V-VI: Amigues 2017

Historia plantarum: Libri I-II: Amigues 1988 • Libri III-IV: Amigues 1989 • Libri V-VI: Amigues 1993・ Libri VII-VIII: Amigues 2003・ Libro IX: Amigues 2006

Opuscula: De igne: Coutant 1971 - De lapidibus: Eichholz $1965 \bullet$ De lassitudine: Fortenbaugh et al. 2003 • Metaphysica: Gutas 2010 • De odoribus: Eigler \& Wöhrle 1993 - De piscibus: Sharples 1992 • De sensibus: Diels 1879: 499-527 • De signis ventorum: 
Sider \& Brunschön 2007 • De sudoribus: Fortenbaugh et al. $2003 \bullet$ De ventis: Coutant \& Eichenlaub 1975 e Mayhew 2018 • De vertigine: Fortenbaugh et al. 2003 • Frammenti: Fortenbaugh et al. 1993

Per le citazioni di autori antichi e bizantini, salvo ove diversamente indicato, si è fatto riferimento alle edizioni utilizzate nel TLG (in ogni caso riscontrate sugli originali). Per le citazioni latine si è invece fatto ricorso alla banca-dati Bibliotheca Teubneriana Latina Online (BTL).

Alla base delle collazioni qui pubblicate è l'edizione bekkeriana del 1831. Poiché molte delle lezioni accolte da Bekker si sono rivelate erronee, per evitare ricadute nella presentazione del dato testuale si è collocata sempre a sinistra la lezione ritenuta superiore, a destra quella considerata inferiore. Nell'indicare gli estremi di una variante si farà sempre riferimento alla linea di testo dove una parola ha inizio.

\section{Conspectus siglorum}

\section{- Sigla codicum:}

B = Marc. gr. IV, 58

[D = Marc. gr. 216]

$\mathbf{F}=$ Vat. gr. 1302

$\mathbf{T}=$ Londinensis Lambeth Palace 1204

$\mathbf{G}=$ Laur. Plut. 60, 19

$\mathbf{H}=$ Laur. Plut. 86, 3 (pars antiqua)

$\mathbf{P}=$ Hafniensis Fabr. $60-4^{\circ}$

$\mathbf{R}=$ Mediol. Ambr. C 4 sup.

$\boldsymbol{\varphi}=$ Bartholomaei translatio Latina

$\boldsymbol{\alpha}=$ consensus codicum $\mathbf{B}(\mathbf{D}) \operatorname{cum} \boldsymbol{\varphi}$

$\boldsymbol{\beta}=$ cons. FT

$\boldsymbol{\psi}=$ cons. $\boldsymbol{\alpha} \boldsymbol{\beta}$

$\gamma=$ cons. HGPR

$\mathbf{X}=$ cons. GPR

\section{- Codices descripti:}

$\mathbf{A}=$ Marc. gr. $200 \bullet \mathbf{L}=$ Vindob. Phil. gr. $231 \bullet \mathbf{C}=$ Marc. gr. $215 \bullet \mathbf{Q}=$ Ambr. A 174 sup. $\bullet$ $\mathbf{J}=$ Vat. Ottob. gr. $45 \bullet \mathbf{Y}=$ Vat. Ottob. gr. $147 \bullet \mathbf{Z}=$ Escur. 68 V $12 \bullet$ Camb. = Cambridge, 
Univ. Library, Dd IV $16 \bullet \mathbf{K}=$ Vat. Urb. gr. $108 \bullet \mathbf{E}=$ Vat. Palat. gr. $162 \bullet \mathbf{O}=$ Bern. 402

- $\mathbf{M}$ = Leid. Voss. gr. $\mathrm{Q}^{\circ} 25$

$\pi=$ cons. $\mathbf{K E O}$

- Editiones antiquae:

Ald. $=$ Editio princeps Aldina (1497) $\bullet$ Bas $^{\mathbf{1}}=$ Editio Basileensis prima (1531) $\bullet \mathbf{B a s}^{\mathbf{2}}=$ Editio Basileensis secunda (1539) • Bas $^{3}=$ Editio Basileensis tertia (1550) • $\boldsymbol{\varsigma}=$ Editio Henrici Stephani (1557)

- Nomina virorum doctorum:

Apelt $=$ Apelt 1888 $\bullet$ Beckmann $=$ Beckmann 1786 $\bullet$ Bk $=$ Bekker $1831 \bullet$ Buss. (emaker) $=$ Bussemaker $1857 \bullet$ Casaubon $=$ Editio Isaacii Casauboni (1590) $\bullet$ Geffcken $=$ Geffcken $1892 \bullet$ Gessner = apud Beckmann $1786 \bullet$ Giann.(ini) = Giannini $1965 \bullet$ Heyne $=$ apud Beckmann $1786 \bullet$ Lucarini = Lucarini $2003 \bullet$ Matthiae $=$ apud Beckmann $1786 \bullet$ Niclas = apud Beckmann 1786 • Salmasius = apud Beckmann 1786 • Sylb.(urg) = Sylburg 1587 - Steph.(anus) = "appendix critica” apud Stephanum 1557 - Wil.(amowitz) = apud Geffcken 1892. 
